\title{
EFFECTS OF THE SECRETORY/EXCRETORY PRODUCTS OF LEPEOPHTHEIRUS SALMONIS ON ATLANTIC SALMON LEUKOCYTE MIGRATION
}

\author{
Jessica Piesz $^{1 *}$, Sarah Barker ${ }^{2 *}$, and Ian BricknelI ${ }^{1 \xi}$ \\ ${ }^{1}$ Aquaculture Research Institute, University of Maine, Orono, Maine 04469 \\ ${ }^{2}$ Fish Vet Group, 350 Commercial Street, Portland, ME 04101
}

\begin{abstract}
Lepeophtheirus salmonis evades its host, Atlantic salmon, by inhibiting inflammatory responses; however, the mechanisms of this activity are not well characterized. Studies from other arthropod ectoparasites have identified chemokine-binding proteins in the saliva and salivary gland extracts that inhibit neutrophil recruitment and activation in vitro and in vivo by binding to chemokines such as leukotriene B4 (LTB4). To determine if L. salmonis is using a similar mechanism to evade the host's immune response the effects of $L$. salmonis secretory/excretory products (SEPs) on LTB4 stimulated leukocytes in vitro were examined. Results showed an increase in leukocyte recruitment in response to LTB4 immune stimulation; yet, pre-incubation of LTB4 with SEPs for 1 hour significantly reduced cell migration compared to LTB4 stimulation alone. This data suggests that L. salmonis inhibits inflammatory responses in Atlantic salmon by secreting proteins with chemokine inhibiting activity. Furthermore, we measured the effects of SEPs on the host's response to chitin, a component of the exoskeleton of arthropods known to induce inflammation and IL-8 production, in vivo. Histological analysis of epithelial tissues collected 6 hours post chitin exposure showed a significant increase in leukocyte recruitment to chitin + dopamine seawater (CDSW) treatment compared to the no injection control. SEPs + chitin and SEPs only treatments were similar, with fewer leukocytes present at injections sites. These treatments were not significantly different from positive controls. To gain a better understanding of the chemotactic pathways disrupted by SEPs, the expression of genes involved in neutrophil activation and cellular chemotaxis in epithelial tissues collected from the in vivo chitin challenge was assessed. Data showed CDSW significantly upregulated IL-8 gene expression relative to the vehicle control at $24 \mathrm{~h}$ post challenge. When SEPs were administered in combination with chitin, IL- 8 expression was not significantly different from the CDSW and chitin only treatments. Results suggest SEPs are not inhibiting IL-8 transcription in response to chitin. While histologically fewer leukocytes are migrating in the chitin + SEP and SEP only treatments, transcriptionally it was shown there is no change in IL-8 expression between chitin and chitin + SEP treatments. The in vivo data suggests SEPs are directly interacting with leukocytes inhibiting the release of IL-8, or are directly interacting with IL-8 inhibiting its ability to interact with immune cells and stimulate an inflammatory response in Atlantic salmon.
\end{abstract}

\author{
KEYWORDS \\ Parasite, leukocyte, SEPs, cytokine, immune evasion \\ "These authors have contributed equally to this work. \\ ECorresponding author. Tel: +1 2075814380. \\ E-mail address: ian.bricknell@maine.edu
}

